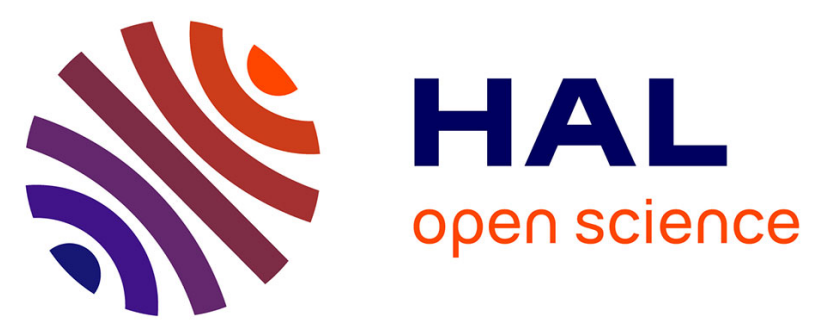

\title{
Cancers du col utérin de stade IB2, IIA et IIB sans extension ganglionnaire traités par chimioradiothérapie préopératoire
}

Laurie Monnier, Emmanuel Touboul, Emile Daraï, Jean Pierre Lefranc, Betty Lauratet, Marcos Ballester, Florence Huguet

\section{To cite this version:}

Laurie Monnier, Emmanuel Touboul, Emile Daraï, Jean Pierre Lefranc, Betty Lauratet, et al.. Cancers du col utérin de stade IB2, IIA et IIB sans extension ganglionnaire traités par chimioradiothérapie préopératoire. Bulletin du Cancer, 2016, 103 (2), pp.164-172. 10.1016/j.bulcan.2015.11.010 . hal01314364

\section{HAL Id: hal-01314364 https://hal.sorbonne-universite.fr/hal-01314364}

Submitted on 11 May 2016

HAL is a multi-disciplinary open access archive for the deposit and dissemination of scientific research documents, whether they are published or not. The documents may come from teaching and research institutions in France or abroad, or from public or private research centers.
L'archive ouverte pluridisciplinaire HAL, est destinée au dépôt et à la diffusion de documents scientifiques de niveau recherche, publiés ou non, émanant des établissements d'enseignement et de recherche français ou étrangers, des laboratoires publics ou privés. 
CANCERS DU COL UTERIN DE STADE IB2, IIA ET IIB SANS EXTENSION

GANGLIONNAIRE TRAITÉS PAR CHIMIORADIOTHERAPIE PREOPERATOIRE

Stage IB2, IIA and IIB cervical carcinoma without lymph node extension treated with neoadjuvant chemoradiotherapy

Monnier L ${ }^{\mathrm{a}, \mathrm{b}}$, Touboul $\mathrm{E}^{\mathrm{a}, \mathrm{b}}$, Daraï $\mathrm{E}^{\mathrm{b}, \mathrm{c}}$, Lefranc JP ${ }^{\mathrm{b}, \mathrm{d}}$, Lauratet $\mathrm{B}^{\mathrm{b}, \mathrm{d}}$, Ballester $\mathrm{M}^{\mathrm{b}, \mathrm{c}}$, Huguet $\mathrm{F}^{\mathrm{a}, \mathrm{b}}$.

a Service d'Oncologie Radiothérapie, Hôpital Tenon, Hôpitaux Universitaires Est Parisien, Paris; ${ }^{\mathrm{b}}$ Faculté de Médecine Pierre et Marie Curie, Université Paris VI; ${ }^{\mathrm{c}}$ Service de Gynécologie Obstétrique, Hôpital Tenon, Hôpitaux Universitaires Est Parisien, Paris; d Service de Chirurgie Gynécologique et Mammaire, Hôpital Pitié-Salpêtrière, Groupe Hospitalier La Pitié Salpêtrière-Charles Foix, Paris.

Correspondant: Dr Laurie MONNIER, Service d'Oncologie Radiothérapie, Hôpital Tenon, 4 rue de la Chine, 75020 Paris; tel 01560166 29; fax 0156016400.

laurie.monnier@tnn.aphp.fr

Titre courant : Chimioradiothérapie et cancer du col utérin. 


\section{RÉSUMÉ}

Objectif de l'étude: Evaluation rétrospective des résultats d'une chimioradiothérapie préopératoire à dose adaptée pour les carcinomes du col utérin de gros volume opérables sans extension ganglionnaire après stadification chirurgicale.

Patients et méthodes : Entre 2000 et 2010, 45 patientes atteintes d'un cancer du col utérin de stade IB2 (11 patientes), IIA2 (3 patientes) et IIB proximal (31 patientes) ont reçu une chimioradiothérapie préopératoire à une dose de 40,50 Gy avec une chimiothérapie concomitante par platine (44 patientes) ou mitomycine (une patiente). Quarante-deux patientes ont eu une curiethérapie utérovaginale à bas débit de dose de 20 Gy. Toutes les patientes ont eu une colpohystérectomie. Trois patientes ont eu une curiethérapie vaginale à bas débit de dose post-opératoire de 20 Gy. Le recul médian était de 34 mois.

Résultats : Seize patientes $(35,6 \%)$ présentaient un résidu tumoral histologique cervical. Six patientes $(13,3 \%)$ ont présenté une rechute tumorale avec un délai médian de 8 mois. Les taux de survie globale et de survie sans rechute à 5 ans étaient respectivement de 88,4\% et 84,7\%. Après analyse univariée, seule la présence d'un résidu tumoral était un facteur prédictif de survie globale $(\mathrm{p}=0,03)$. Une toxicité tardive a été observée chez 7 patientes.

Conclusion : Une chimioradiothérapie suivie d'une chirurgie d'exérèse de type Piver II dans les cancers du col utérin de stade I et II de gros volume opérables sans extension ganglionnaire après stadification chirurgicale préthérapeutique permet d'obtenir un bon contrôle tumoral local avec un taux de survie à 5 ans élevé.

Mots clés : cancer du col utérin de gros volume; chimioradiothérapie préopératoire ; hystérectomie ; curiethérapie. 


\section{ABSTRACT}

Purpose: To evaluate the results of preoperative chemoradiation for resectable bulky cervical carcinoma without lymph node involvement after surgical lymph node staging.

Patients and methods: Between 2000 and 2010, 45 patients with cervical carcinoma stage IB2 (11 patients), IIA2 (3 patients) and IIB with proximal parametrial invasion (31 patients) were treated with pelvic radiation therapy at a dose of 40.5 Gy and concurrent platin (44 patients) or mitomycin (one patient). Forty-two patients had low-dose-rate pre-operative uterovaginal brachytherapy at a dose of $20 \mathrm{~Gy}$. All patients underwent hysterectomy. Three patients had post-operative low-dose-rate vaginal brachytherapy at a dose of $20 \mathrm{~Gy}$. The median follow up was 34 months.

Results: A pathologic cervical residual tumor was observed in 16 patients (35.6\%). Six patients presented a relapse $(13.3 \%)$ with a median delay of 8 months. The 5 -year overall survival and disease free survival rates were $88.4 \%$ and $84.7 \%$, respectively. In univariable analysis, a cervical residual tumor was the only predictive factor of overall survival $(\mathrm{p}=0.03)$. Late toxicity was observed in seven patients.

Conclusion: Chemoradiation followed by surgery for resectable bulky stage I-II cervical carcinoma without lymph node involvement on pretreatment surgical staging can be used with a good local control and a high rate of 5-year overall survival.

Key words: Bulky cervical carcinoma; pre-operative chemoradiotherapy; hysterectomy; brachytherapy. 


\section{Introduction}

Les cancers du col utérin localisés de gros volume $(\geq 4 \mathrm{~cm}$ de plus grand diamètre) de stade IB2 et IIA2 selon la classification de FIGO 2009 «International Federation of Gynecology and Obstetric system » [1] et stade IIB proximal selon la classification de l'Institut Gustave Roussy méconu en dehors de la France [2] sont des formes à risque élevé de rechute locorégionale et/ou métastatique [3-5] pour lesquelles une association chimioradiothérapie concomitante à visée curative, suivie ou non d'une chirurgie de rattrapage, est actuellement considérée comme un standard thérapeutique [6,7]. Cependant, quelques institutions ont proposé une association chimioradiothérapie préopératoire à dose adaptée suivie d'une chirurgie programmée, soit de type hystérectomie extrafasciale [8], soit de type hystérectomie élargie de classe II avec ou sans curage ganglionnaire pelvien $[9,10]$ et ceci afin d'essayer d'obtenir un taux de contrôle tumoral élevé avec un risque réduit de complications postthérapeutiques sévères. Nous avons précédemment publié nos résultats après association chimioradiothérapie concomitante préopératoire suivie d'une chirurgie programmée pour des carcinomes du col utérin de gros volume localisés sans extension ganglionnaire pelvienne et/ou lomboaortique visible après stadification clinique et radiologique [11, 12]. Dans ce travail, nous rapportons une série rétrospective de 45 patientes présentant un cancer du col utérin de gros volume localisé, sans extension ganglionnaire après une stadification ganglionnaire chirurgicale per-coelioscopique lomboaortique avec ou sans curage pelvien. Dans notre référentiel, cette association chimioradiothérapie préopératoire ne s'adresse qu'aux patientes sans atteinte ganglionnaire histologique, les patientes présentant une atteinte ganglionnaire étant traitées par association chimioradiothérapie exclusive à dose curative. 


\section{Patientes et méthodes}

\subsection{Caractéristiques des patientes}

Entre octobre 2000 et septembre 2010, des patientes atteintes d'un carcinome du col utérin stade IB2, IIA2 et IIB proximal de plus de 4 centimètres ont reçu une chimioradiothérapie pelvienne et curiethérapie utérovaginale de complément pré-opératoire (quand elle était anatomiquement possible) suivie d'une chirurgie programmée. Elles ont été sélectionnées pour cette étude de manière rétrospective. En effet, les patientes candidates à cette stratégie étaient uniquement celles présentant un carcinome du col utérin de gros volume ( $>4 \mathrm{~cm})$ sans atteinte ganglionnaire histologique. Le bilan pré-thérapeutique comprenait: un examen gynécologique, un examen anatomopathologique (biopsies de l'endocol et de l'exocol, voir conisation si nécessaire), un bilan biologique (hémogramme sanguin, ionogramme sanguin avec créatininémie), une Imagerie par Résonance Magnétique (IRM) pelvienne, un scanner thoraco-abdominopelvien et si c'était nécessaire, une cystoscopie ou une rectoscopie. Certaines patientes ont eu une Tomographie à Emission de Positons (TEP) au 18 $\alpha$-fluorodésoxyglucose. Toutes les patientes ont eu une stadification chirurgicale ganglionnaire préthérapeutique per-coelioscopique. Dans tous les cas, aucun envahissement ganglionnaire histologique n'a été mis en évidence. Aucune des patientes n'avait antérieurement été traitée pour un autre cancer. Les caractéristiques des patientes sont rapportées dans le tableau 1.

\subsection{Traitement}

Le traitement a consisté en une irradiation externe conformationnelle tridimensionnelle pelvienne première avec des photons d'énergie de 15 à $25 \mathrm{MV}$, après simulation virtuelle par scanner, fusion d'images IRM et TEP si celle-ci était effectuée. Toutes les patientes ont reçu une chimiothérapie concomitante à l'irradiation pelvienne. Le protocole de chimiothérapie 
associait le 5 -fluorouracile $\left(800 \mathrm{mg} / \mathrm{m}^{2} /\right.$ jour en perfusion intraveineuse continue sur 4 jours) et du cisplatine $\left(25 \mathrm{mg} / \mathrm{m}^{2} / \mathrm{j}\right.$ en perfusion intraveineuse de $\mathrm{J} 1$ à $\left.\mathrm{J} 4\right)$ la première et la quatrième semaine d'irradiation. La surveillance clinique au cours du traitement était hebdomadaire. Un hémogramme et un ionogramme sanguin avec mesure de la créatininémie ont été réalisés avant chaque cycle de chimiothérapie. L'association radiochimiothérapie concomitante était suivie d'une curiethérapie réalisée avant la chirurgie si elle était techniquement faisable. La planification de la dose était réalisée selon les recommandations du rapport 38 de l'ICRU [13]. Elle était réalisée avec un applicateur de type moule vaginal personnalisé. La planification de la dose était réalisée sur clichés orthogonaux puis sur scanner à partir de 2008. Le chargement était manuel, différé, avec des sources d'Iridium 192. La prescription était de 20 Gy au point A. Une chirurgie de type colpohystérectomie élargie non conservatrice de type Piver II était programmée à la suite de la curiethérapie [14]. La surveillance post thérapeutique a été effectuée tous les 3 mois la première année puis tous les 6 mois au cours des cinq premières années puis annuellement. Les complications tardives ont été évaluées selon la classification CTCAE v4.0 (Common Terminology Criteria for Adverse Events).

\subsection{Analyse statistique}

Les résultats analysés étaient la toxicité aigüe, les complications tardives sévères, la réponse histologique au traitement, le contrôle tumoral et les taux de survie globale et sans rechute. Les proportions et les moyennes ont été comparées, respectivement, par le test du $\chi 2$ et le test t de Student. Les courbes de survie ont été calculées selon la méthode d'analyse statistique de Kaplan et Meier. Le test du log-rank a été utilisé pour l'analyse univariée des facteurs pronostiques de survie. L'analyse multivariée des données de survie a été réalisée en utilisant le modèle de régression logistique de Cox. Une valeur du p inférieure à 0,05 a été considérée 
comme statistiquement significative. Les résultats ont été donnés avec leur écart-type. Les analyses ont été réalisées avec le logiciel Statview v5.

\section{Résultats}

\subsection{Patientes et déroulement du traitement}

45 patientes ont été sélectionnées de manière rétrospectives pour cette étude avec un suivi médian de 34 mois (moyenne : 39 mois ; extrêmes : 6,5-93,5). Toutes les patientes avaient un indice de performance selon la classification OMS (Organisation Mondiale de la Santé) $\leq 1$. Une TEP a finalement été effectuée chez 17 patientes. La stadification ganglionnaire chirurgicale préthérapeutique était lomboaortique rétropéritonéale chez 11 patientes et à la fois lomboaortique rétropéritonéale et pelvienne transpéritonéale chez 34 patientes (ceci dû aux différences de pratiques chirurgicales des centres ayant adressés les patientes). Le nombre moyen de ganglions lomboaortiques prélevés était de 16 (extrêmes : 4-39) et le nombre moyen de ganglions iliaques prélevés était de 6 à gauche (2-12) et 6 à droite (0-12). La dose médiane délivrée dans le volume tumoral, les volumes ganglionnaires pelviens (iliaques primitifs, externes, internes, présacrés), les paramètres et le tiers supérieur du vagin était de 40,5 Gy en 18 fractions et 30 jours (soit 41,3 Gy en EQD2 : «Equivalent Dose in 2 gy Fraction ", c'est à dire l'équivalent de dose pour un fractionnement classique de 2 Gy par séance). Les EQD2 ont été calculés avec un rapport $\alpha / \beta$ de 10 pour la tumeur (col, paramètres, vagin), 5 pour le rectum et 2 pour la vessie. 42 patientes ont reçu le protocole de chimiothérapie initialement proposé, associant le 5FU et le cisplatine. Une patiente a reçu du cisplatine hebdomadaire à la dose de $40 \mathrm{mg} / \mathrm{m}^{2}$. En raison d'une insuffisance rénale modérée, le cisplatine a été remplacé par du carboplatine AUC 6 à J1 et J29 chez une patiente et par de la mitomycine $\mathrm{C}$ à la dose de $8 \mathrm{mg} / \mathrm{m}^{2}$ à $\mathrm{J} 1$ et $\mathrm{J} 29$ chez une autre patiente. Deux patientes 
n'ont eu qu'un seul cycle de chimiothérapie en raison d'une mauvaise tolérance digestive et hématologique. Quarante-deux patientes ont eu une curiethérapie utérovaginale de complément préopératoire à bas débit de dose continu après un délai médian de 14 jours (moyenne : 15 jours, extrêmes : 6-32 jours) et avec un débit de Kerma linéique dans l'air moyen de $24 \mu \mathrm{Gy} \cdot \mathrm{h}^{-1} \cdot \mathrm{m}^{2} \cdot \mathrm{cm}^{-1}$ (extrêmes : 19,17-30). La dose au point A était en moyenne de 1,6 Gy (extrêmes : 6,4-30,4Gy). La dose délivrée sur l'isodose de référence était de 20 Gy pour toutes les patientes, avec un débit de dose médian de 0,5 Gy/heure (extrêmes : 0,460,83). La dose moyenne au point vessie et au point rectum (EQD2) était respectivement de 11,5 Gy (extrêmes : 4,8-28,3 Gy) et 16,7 (extrêmes : 5,6-21,8Gy). Toutes les patientes ont eu une colpohystérectomie élargie non conservatrice de type Piver II. Un curage ganglionnaire pelvien a été réalisé chez les 11 patientes qui n'en avaient pas eu lors de la stadification ganglionnaire initiale. Le délai médian entre la fin de la radiothérapie et la procédure chirurgicale était de 43 jours (moyenne : 44, extrêmes : 27-108). Trois patientes ont eu une curiethérapie vaginale postopératoire à bas débit de dose, à l'aide d'un moule vaginal personnalisé et de sources d'Iridium 192, à la dose de 20 Gy prescrite à $5 \mathrm{~mm}$ des parois de l'applicateur. Pour ces trois patientes, les conditions anatomiques locales après chimioradiothérapie ne permettaient pas de réaliser une curiethérapie utérovaginale (anatomie cervico-vaginale remaniée, destruction du col et sténose cervicale infranchissable).

\subsection{Tolérance}

Après stadification ganglionnaire chirurgicale pré-thérapeutique, nous avons observé une complication postopératoire chez 9 patientes (20\%) avec 8 lymphocèles (3/11 en cas de curage ganglionnaire lomboaortique seul et 5/34 en cas de curage pelvien associé au lomboaortique) et une plaie de la veine rénale (dans le groupe curage pelvien et lomboaortique). 
Après la colpohystérectomie élargie, 21 patientes (46,5\%) ont présenté une ou plusieurs complications postopératoires. Ces complications sont détaillées dans le tableau 2. La complication la plus fréquente était un œdème proximal modéré d'un membre inférieur observé chez 7 patientes $(15,5 \%)$ dont $3(9 \%)$ après curage ganglionnaire pelvien réalisé avant chimioradiothérapie et $4(36 \%)$ après curage pelvien réalisé après chimioradiothérapie. Quatre d'entre eux ont été régressifs.

Au cours de la chimioradiothérapie préopératoire, une toxicité aigüe hématologique sévère de grade 3 selon la classification CTCAE v4.0 a été rapportée dans neuf cas (20\%) : leucopénie de grade 3 dans neuf cas, associée à une anémie de grade 3 dans quatre cas, à une thrombopénie de grade 3 dans quatre cas et à une thrombopénie de grade 4 dans un cas. De plus, nous avons observé une toxicité aigüe de grade 3 digestive dans huit cas (17,8\%), vésicale dans deux cas $(4,4 \%)$ et cutanéo-muqueuse périnéale dans un cas $(2,2 \%)$. Il n’a pas été observé de toxicité de grade 4 ni de décès lié au traitement.

Des complications tardives post-radiques de grade 3 selon la classification CTCAE v4.0 ont été observées chez sept patientes (15,5\%) et sont rapportées dans le tableau 3.

Aucun des facteurs étudiés tels que l'âge, la taille tumorale, le stade FIGO, la chronologie de la curiethérapie, la chronologie du curage ganglionnaire pelvien, n'ont significativement influencé le risque de complications après colpohystérectomie élargie ou le risque de complications post-radiques tardives de grade 3.

\subsection{Réponse histologique}

Après analyse anatomopathologique des pièces opératoires, aucun résidu tumoral n'a été observé dans 29 cas $(64,4 \%)$. Un reliquat tumoral cervical persistait chez 16 patientes $(35,6 \%)$. Il était $\leq 5 \mathrm{~mm}$ dans six cas $(37,5 \%$ des cas de reliquats) et $>5 \mathrm{~mm}$ dans dix cas (62,5\%). La taille médiane du résidu tumoral cervical était de $8 \mathrm{~mm}$ (moyenne 10,5 $\pm 2 \mathrm{~mm}$; 
extrêmes : 1-30). La collerette vaginale était envahie avec une recoupe histologiquement saine dans deux cas $(4,4 \%)$. Un envahissement paramétrial a été observé dans trois cas $(6,6 \%)$. Les ganglions prélevés lors des 11 curages pelviens effectués après chimioradiothérapie étaient indemnes de tumeur. Aucun des facteurs étudiés tels que la taille tumorale, le type histologique, la différenciation, le stade FIGO, la chronologie de la curiethérapie n'a significativement influencé le taux de réponse histologique complète.

\subsection{Contrôle tumoral}

Six patientes avaient présenté une rechute tumorale au moment de l'analyse. Le délai médian de survenue de la rechute tumorale était de 8 mois (moyenne : 11 mois ; extrêmes : 7,5-27 mois). La répartition topographique des rechutes était la suivante: une rechute locale vaginale, deux rechutes métastatiques isolées, une rechute à la fois locale, latéro-pelvienne et métastatique et deux rechutes à la fois lomboaortique et métastatique. Le taux de contrôle était de $96 \%$ et le taux de contrôle à distance de $89 \%$. La médiane de survie après le diagnostic de rechute était de 6 mois (extrêmes : 2 à 14 mois). Aucun des paramètres étudiés tels la taille tumorale, le type histologique, la différenciation, le stade FIGO, la présence d'un reliquat tumoral histologique n'ont eu un impact significatif sur la survenue d'une rechute tumorale. Cependant, une rechute tumorale pelvienne et/ou extra pelvienne a été observée dans 4 cas sur $16(25 \%)$ en présence d'un résidu tumoral histologique contre 2 cas sur 29 (7\%) en 1'absence de résidu tumoral. Concernant la taille tumorale, une rechute tumorale a été observée dans 3 cas sur $10(33 \%)$ en présence d'un résidu tumoral $>5 \mathrm{~mm}$ contre 3 cas sur $35(8,5 \%)$ pour un résidu $\leq 5 \mathrm{~mm}$. 


\subsection{Survie}

Le recul médian était de 34 mois (moyenne : 39 mois; extrêmes : 6,5-93,5). Au moment de l'analyse, cinq patientes étaient décédées de leur cancer. Les taux de survie globale à deux et cinq ans étaient respectivement de $90,6 \% \pm 4,5$ et $87,8 \% \pm 5$. Après analyse univariée, la réponse histologique complète était le seul facteur prédictif de survie globale à 2 ans : 96,3\% en cas de réponse histologique complète contre $81,3 \%$ en cas de résidu tumoral histologique, $(\mathrm{p}=0,03)$ (tableau 4). Au moment de l'analyse, 6 patientes avaient une rechute tumorale. Les taux de survie sans rechute à deux et cinq ans étaient respectivement de $88,4 \% \pm 5$ et $84,7 \% \pm$ 6. Nous n'avons pas identifié de facteur prédictif de rechute au moment de l'analyse univariée.

\section{Discussion}

Bien que la nature rétrospective de ce travail rende la comparaison difficile, il semble que nos résultats en termes de survie sans rechute à 2 et 5 ans, respectivement de $88,4 \%$ et $84,7 \%$, soient légèrement supérieurs à ceux que nous avions rapportés dans deux publications antérieures pour des patientes atteintes d'un cancer du col utérin avec des stades FIGO comparables et traitées avec le même protocole de chimioradiothérapie [11, 12]. Dans ces deux publications antérieures, les taux de survie sans rechute à 2 et 5 ans étaient respectivement de $80 \%$ et $71 \%$ [11] et de $80 \%$ et $72 \%$ [12]. Cette différence est probablement liée à la réalisation ou non d'une stadification ganglionnaire chirurgicale pré thérapeutique qui reste, actuellement, le meilleur moyen pour déterminer l'absence d'extension tumorale ganglionnaire dans les territoires explorés [15-17]. En effet, elle a été effectuée dans tous les cas dans cette série, alors qu'elle n'avait pas été réalisée dans la première série de 45 patientes antérieurement publiée [11]et dans seulement $13 \%$ des cas (12/92) dans la deuxième série [12]. Actuellement, dans notre référentiel, la 
chimioradiothérapie à dose préopératoire suivie d'une chirurgie centropelvienne programmée n'est réservée qu'aux tumeurs de stade IB2, IIA2 et IIB proximal dont la stadification ganglionnaire pré thérapeutique (TEP au $18 \alpha$ FDG puis chirurgicale) confirme l'absence d'extension tumorale ganglionnaire [18]. Dans notre série, le taux de résidu tumoral histologique centropelvien après chimioradiothérapie pelvienne à dose modérée pré opératoire de 40,5 Gy en 4,5 semaines et curiethérapie utéro-vaginale de complément à bas débit de dose de 20 Gy était de 35,6\%. Cependant, nous n'avons observé que deux rechutes tumorales locorégionales $(4,4 \%)$, dont une associée à une rechute ganglionnaire pelvienne et métastatique. Dans une série de l'Institut Curie avec un protocole de chimioradiothérapie pelvienne préopératoire voisin à la dose de 36 Gy en 22 séances (1,8 Gy par séance, 5 séances par semaine) suivie d'une curiethérapie utéro-vaginale de complément à bas débit de dose de 24 Gy pour des tumeurs classées IB2, IIA2 et IIB proximal, le taux de résidu histologique était de 44\% (31/70) et le taux actuariel de rechute locorégionale à 5 ans n'était que de $13 \%$ [10]. Dans notre série, comme dans celle de l'Institut Curie, la chirurgie programmée était réalisée 5 à 6 semaines après la curiethérapie utéro-vaginale de complément et correspondait dans la majorité des cas à une colpohystérectomie élargie de type Piver II, avec un taux de toxicité tardive sévère réduite, dominée par le lymphœdème du membre inférieur non régressif, dans $8,8 \%$ pour notre série et dans $3 \%$ pour celle de l'Institut Curie [10]. Cependant, la stratégie optimale et la place de la chirurgie reste discutée dans les carcinomes du col utérin stade IB2, IIA2, IIB proximaux sans atteinte ganglionnaire initiale. Quelques institutions ont rapporté des résultats comparables à notre série, après une chimioradiothérapie pelvienne préopératoire à une dose plus élevée de 45 à 50 Gy en 5 à 6 semaines, suivie d'une curiethérapie utéro vaginale de complément. La chirurgie programmée, effectuée 6 semaines après curiethérapie, était, le plus souvent, limitée à une hystérectomie totale de type Piver I et parfois si nécessaire élargie de type Piver II ou III. Les taux de réponse histologique complète 
étaient compris entre $6 \%$ et $35 \%$ selon l'importance du volume tumoral centropelvien et la dose d'irradiation délivrée [8, 19-21]. Les taux de rechute locorégionale étaient compris entre $15 \%$ et $27 \%$ selon les séries et les taux de complications sévères tardives restaient relativement bas après hystérectomie extra fasciale de type Piver I, inférieur à $1 \%[8,9,20$, 21]. Ce taux augmentait significativement lorsque la chirurgie était élargie de type Piver II ou III [20-21]. Par ailleurs, la curiethérapie utéro-vaginale de complément préopératoire, quand elle était réalisable, augmentait significativement le taux de réponse histologique complète [21]. Le tableau 5 résume les principales séries d'association radiochimiothérapie avant chirurgie de clôture. Ces dernières années, la plupart des référentiels ou des recommandations proposent une chimioradiothérapie exclusive à visée curative avec des doses d'irradiation plus élevées, comprenant une irradiation externe conformationnelle pelvienne à une dose de 45 à 50 Gy en 5 à 6 semaines, associée à une chimiothérapie concomitante avec du cisplatine ou de la mitomycine et une curiethérapie utéro-vaginale de complément $[6,22]$. Les résultats en termes de survie sont comparables à ceux rapportés avec la stratégie thérapeutique préopératoire et chirurgicale, avec des taux de rechute locorégionales compris entre $13 \%$ et $22 \%$ selon les séries $[6,23]$. Une chirurgie de rattrapage n'est alors proposée que pour certaines patientes, en cas de persistance d'un reliquat tumoral centropelvien ou en cas de rechute locale, en l'absence d'évolution ganglionnaire ou métastatique [24]. Le taux de contrôle locorégional après chirurgie de rattrapage est de l'ordre de 30 à $50 \%$ avec un taux de complications sévères de l'ordre de $10 \%$ après hystérectomie extra-fasciale et de 30 à $35 \%$ en cas de chirurgie élargie de type Piver II ou II ou si nécessité d'exentération. En cas de chirurgie élargie, le taux de survie à 5 ans est de 20 à $40 \%$ et le taux de mortalité postopératoire est de $10 \%$ [24]. Le diagnostic de maladie résiduelle ou de rechute précoce localisée centropelvienne après chimioradiothérapie exclusive est parfois difficile, les examens complémentaires de type IRM pelvienne, TEP au 18 $\alpha$ FDG et biopsies dirigées, 8 à 
10 semaines après le traitement ne sont pas sensibles et spécifiques à 100\% [24,26]. L'étude multicentrique française ERRICC, en cours, étudie la corrélation entre l'imagerie après chimioradiothérapie et curiethérapie (IRM de diffusion et TEP) et le résultat histologique après hystérectomie pour évaluer l'apport de ces techniques dans l'évaluation de la réponse tumorale pour les tumeurs de stade IB2, IIA2 et IIB proximal. Bien que le pronostic d'une rechute tumorale après une chimioradiothérapie à visée curative reste globalement médiocre, avec un taux de survie à 5 ans, toutes topographies de la rechute confondues de $10 \%$ [13], l'intérêt d'une chirurgie adjuvante systématique après chimioradiothérapie à dose curative, n’est actuellement pas démontré. Une étude prospective randomisée de phase III a inclus des patientes atteintes d'un cancer du col utérin de stade IB2 traitées par radiothérapie externe pelvienne et curiethérapie utéro-vaginale [27]. Cette étude a comparé un groupe de 124 patientes irradiées jusqu'à la dose de 80 Gy (dose au point $\mathrm{A}$ de Manchester) et un groupe de 132 patientes irradiées jusqu'à la dose de 75 Gy (point A) puis hystérectomie extra-fasciale de type Piver I. Le taux de rechute locorégionale à 5 ans était significativement plus bas dans le groupe avec chirurgie ( $27 \%$ sans hystérectomie contre $15 \%$ avec) et la survie sans rechute était de $53 \%$ sans hystérectomie et $62 \%$ avec $(\mathrm{p}=0,04)$. Cependant, l'hystérectomie n'avait pas d'impact significatif sur la survie globale. Une étude prospective randomisée de phase III menée par la Fédération Nationales des Centres de Lutte contre le Cancer, GYNECO 02, a évalué l'impact d'une hystérectomie de type Piver I ou II 6 à 8 semaines après une chimioradiothérapie chez des patientes en apparente réponse complète après évaluation clinique et radiologique [7]. Les patientes avaient un cancer du col utérin de stade IB2 et II et recevaient une radiothérapie pelvienne de 45 à 50 Gy, à raison de 2 Gy par séance, 5 séances par semaine, suivie après un intervalle de 1 à 2 semaines d'une curiethérapie utéro-vaginale de 15 Gy à bas débit de dose. La chimiothérapie concomitante comprenait 6 cycles de cisplatine hebdomadaire à la dose de $40 \mathrm{mg} / \mathrm{m}^{2}$, le sixième cycle étant effectué pendant la 
curiethérapie [7]. Les taux de survie sans rechute et de survie globale à 3 ans n'étaient pas significativement différents, respectivement de $72 \%$ et $86 \%$ en cas de chirurgie contre $89 \%$ et 97\% en l'absence de chirurgie. Les auteurs concluaient que les résultats de l'étude suggèrent l'absence d'impact de la chirurgie en cas de réponse complète clinique et radiologique. Cependant, l'étude a été interrompue précocement par manque d'inclusion. De plus, 18 patientes avaient des images suspectes d'envahissement ganglionnaire pelvien lors du bilan d'extension initial et seules 13 patientes ont eu une stadification ganglionnaire lomboaortique chirurgicale. Par conséquent, la stratégie thérapeutique des carcinomes du col utérin opérables de stade IB2, IIA2 et IIB proximal sans extension ganglionnaire après stadification initiale reste un sujet de controverse. Aucune étude prospective randomisée de phase III concernant ces formes n'a comparé une chimioradiothérapie préopératoire (dose modérée ou plus élevée) suivie d'une chirurgie de type I ou II et une chimioradiothérapie exclusive avec chirurgie de rattrapage uniquement en cas de résidu tumoral. Comme notre série, quelques études ont montré que la persistance d'un résidu tumoral histologique à l'examen de la pièce opératoire lors d'une chirurgie adjuvante après chimioradiothérapie préopératoire était un facteur de risque significatif de rechute tumorale [18,21]. L'intensification du traitement préopératoire avec l'ajout d'une thérapie ciblée comme le bevacizumab pourrait faire l'objet d'une étude prospective. En effet, l'ajout du bevacizumab à la chimiothérapie en première ligne métastatique a montré son intérêt en termes de contrôle local et de survie [28]. Les techniques d'irradiation conformationnelle avec modulation d'intensité (RCMI) sont également en cours d'évaluation dans ce domaine [29, 30]. L'association de la RCMI et de la curiethérapie utérovaginale guidée par l'image devrait améliorer la tolérance de la chimioradiothérapie préopératoire en diminuant la toxicité aigüe intestinale et hématologique ainsi que la toxicité tardive osseuse, intestinale et vésicale en diminuant la dose aux organes sains de voisinage 
[31]. Enfin, la recherche de facteurs biologiques prédictifs de la réponse tumorale à la radiothérapie pourrait mieux guider l'indication d'une chirurgie [32].

\section{Conclusion}

La combinaison d'une chimioradiothérapie pelvienne à dose modérée préopératoire suivie d'une curiethérapie utéro-vaginale de complément avec une chirurgie de type colpohystérectomie non conservatrice Piver I ou II pour un carcinome du col utérin de stade IB2, IIA2 et IIB proximal, sans extension ganglionnaire après stadification ganglionnaire chirurgicale permet d'obtenir un bon contrôle tumoral locorégional et un taux de survie à 5 ans élevé. Ces résultats pourraient être améliorés par le développement des techniques de RCMI, la recherche de marqueurs biologiques prédictifs de la réponse tumorale après radiothérapie et l'évaluation de l'apport d'une thérapie ciblée.

Conflits d'intérêt : F.H. Conférences : invitations en qualité d'intervenant pour Merck Serono et Roche. 


\begin{tabular}{|c|c|c|}
\hline Caractéristiques & $\mathrm{n}=45$ & $\%$ \\
\hline \multicolumn{3}{|l|}{ Age (ans) } \\
\hline moyenne & $49,4 \pm 1,5$ & \\
\hline médiane & 47 & \\
\hline extrêmes & $25-77$ & \\
\hline \multicolumn{3}{|l|}{ Stade FIGO } \\
\hline IB2 & 11 & 24,4 \\
\hline IIA2 & 3 & 6,5 \\
\hline IIB proximal & 31 & 69 \\
\hline \multicolumn{3}{|c|}{ Taille tumorale $(\mathrm{cm})$} \\
\hline moyenne & $4.9 \pm 0,1$ & \\
\hline médiane & 5 & \\
\hline extrêmes & $2.5-7.3$ & \\
\hline \multicolumn{3}{|l|}{ Histologie $(\mathrm{n} ; \%)$} \\
\hline C.Epidermoïde & 34 & 75.5 \\
\hline Adenocarcinome & 10 & 22.5 \\
\hline Neuroendocrine & 1 & 2 \\
\hline \multicolumn{3}{|c|}{ Différenciation (n ;\%) } \\
\hline Bien différenciés & 17 & 37,5 \\
\hline Moyennement diff & 15 & 33,5 \\
\hline Peu différenciés & 10 & 22,5 \\
\hline NA & 3 & 6,5 \\
\hline \multicolumn{3}{|c|}{ Emboles tumorales $(\mathrm{n} ; \%)$} \\
\hline Oui & 4 & 9 \\
\hline Non & 24 & 53,5 \\
\hline NA & 17 & 37,5 \\
\hline \multicolumn{3}{|l|}{$\mathbf{S C C}(\mathrm{ng} / \mathrm{ml}) *$} \\
\hline$\leq 1,5$ & 15 & 44 \\
\hline$>1,5$ & 18 & 53 \\
\hline NA & 1 & 3 \\
\hline
\end{tabular}

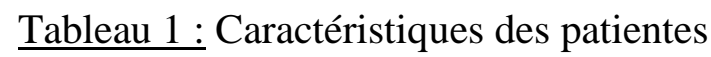

FIGO : Fédération Internationale des Gynécologues et Obstétriciens.

* : Sur 34 carcinomes épidermoïdes 


\begin{tabular}{|c|c|c|c|c|c|c|}
\hline \multirow[b]{2}{*}{ Complications } & \multicolumn{2}{|c|}{$\begin{array}{c}\text { Groupe I } \\
n=34\end{array}$} & \multicolumn{2}{|c|}{$\begin{array}{c}\text { Groupe II } \\
n=11\end{array}$} & \multicolumn{2}{|c|}{$\begin{array}{l}\text { Total } \\
n=45\end{array}$} \\
\hline & $\mathrm{n}$ & $(\%)$ & $\mathrm{n}$ & $(\%)$ & $\mathrm{n}$ & $(\%)$ \\
\hline $\begin{array}{l}\text { Phlébite d'un membre } \\
\text { inferieur }\end{array}$ & 1 & (3) & - & - & 1 & (7) \\
\hline $\begin{array}{l}\text { Edème modéré d'un } \\
\text { membre inférieur }\end{array}$ & 3 & (9) & 4 & (36) & $7 *$ & $(15,5)$ \\
\hline Lyphocèle pelvien & - & - & 4 & (36) & 4 & (9) \\
\hline Hématome pelvien & 1 & (3) & - & - & 1 & (2) \\
\hline Désunion cicatrice & 2 & (6) & - & - & 2 & $(4,5)$ \\
\hline $\begin{array}{l}\text { Urétéro-hydronéphrose } \\
\text { modérée }\end{array}$ & 5 & $(14,5)$ & 2 & (19) & 7 & (15) \\
\hline Abcès de paroi & 1 & (3) & - & - & 1 & (2) \\
\hline Fistule vésico-vaginale & 1 & (3) & - & - & 1 & (2) \\
\hline Brèche vésicale & 2 & (6) & - & - & 2 & $(4,5)$ \\
\hline $\begin{array}{l}\text { Incontinence urinaire } \\
\text { d'effort }\end{array}$ & 3 & (9) & 1 & (9) & 4 & (9) \\
\hline $\begin{array}{l}\text { Nombre de patiente } \\
\text { ayant eu } 1 \text { ou plusieurs } \\
\text { complications }\end{array}$ & 15 & (44) & 6 & (54) & 21 & $(46,5)$ \\
\hline
\end{tabular}

$\underline{\text { Tableau } 2}$ : Complications post opératoires après colpohystéréctomie élargie.

Groupe I : Stadification ganglionnaire chirurgicale première à la fois pelvienne et lombo aortique.

Groupe II : Stadification ganglionnaire chirurgicale première uniquement lomboaortique.

*4/7 ont été regressifs 


\begin{tabular}{|c|c|c|}
\hline Complications & $\mathrm{n}=45$ & $\%$ \\
\hline Grêle/Colon & 1 & 2 \\
\hline Rectum & 2 & 4,5 \\
\hline Vessie & 3 & 6,5 \\
\hline Vagin & 1 & 2 \\
\hline Os & 1 & 2 \\
\hline Fibrose pelvienne & 1 & 1 \\
\hline $\begin{array}{l}\text { Nombre de patientes } \\
\text { ayant eu au moins une } \\
\text { complication tardive } \\
\text { sévère de grade } 3\end{array}$ & 7 & 15,5 \\
\hline
\end{tabular}

$\underline{\text { Tableau } 3}$ : Complications post radiques tardives selon le score SOMA-LENT de grade 3 


\begin{tabular}{|c|c|c|c|c|c|}
\hline Paramètres & $\mathbf{n}$ & n décès & $\begin{array}{c}\text { SG } 2 \text { ans } \\
(\%)\end{array}$ & $\begin{array}{c}\mathbf{n} \\
\text { patientes } \\
\text { à risque }\end{array}$ & $\begin{array}{c}P \\
(\text { logrank) }\end{array}$ \\
\hline \multicolumn{6}{|l|}{ Age } \\
\hline$\leq 45$ ans & 23 & 2 & $95,7 \pm 4$ & 20 & 0,5 \\
\hline$>45$ ans & 22 & 3 & $85 \pm 8$ & 17 & \\
\hline \multicolumn{6}{|l|}{ TailleTumorale } \\
\hline$\leq 4 \mathrm{~cm}$ & 13 & 1 & $92,3 \pm 7$ & 11 & 0,7 \\
\hline$>4 \mathrm{~cm}$ & 32 & 4 & $90 \pm 5$ & 26 & \\
\hline \multicolumn{6}{|l|}{ Histologie } \\
\hline Epidermoïdes & 34 & 5 & $87,5 \pm 6$ & 27 & $\mathrm{NC}$ \\
\hline Non Epidermoïdes & 11 & 0 & 100 & 10 & \\
\hline \multicolumn{6}{|l|}{ Différenciation } \\
\hline Bien différenciés & 17 & 4 & $87 \pm 9$ & 11 & 0,8 \\
\hline Moyennement à peu diff & 25 & 1 & $88 \pm 6$ & 22 & \\
\hline $\mathrm{NA}$ & 3 & 0 & & & \\
\hline \multicolumn{6}{|l|}{ Stade FIGO } \\
\hline IB2-IIA & 14 & 2 & $92,3 \pm 7$ & 12 & 0,8 \\
\hline IIB proximal & 31 & 3 & $89,7 \pm 6$ & 25 & \\
\hline \multicolumn{6}{|l|}{$\begin{array}{l}\text { Taux de SCC sérique } \\
(\mathrm{ng} / \mathrm{ml})^{*}\end{array}$} \\
\hline$\leq 1,5 \mathrm{ng} / \mathrm{ml}$ & 15 & 1 & $87 \pm 9$ & 13 & 0,9 \\
\hline$>1,5 \mathrm{ng} / \mathrm{ml}$ & 18 & 4 & $87 \pm 9$ & 12 & \\
\hline NA & 1 & & & & \\
\hline \multicolumn{6}{|l|}{ Reliquat tumoral } \\
\hline Oui & 16 & 4 & $81,3 \pm 10$ & 11 & 0,03 \\
\hline Non & 29 & 1 & $96,3 \pm 4$ & 26 & \\
\hline Total & 45 & 5 & $87,8 \pm 5$ & 37 & \\
\hline
\end{tabular}

$\underline{\text { Tableau } 4}$ : Facteurs prédictifs de la survie globale, analyse unifactorielle.

SG : survie globale ; SCC : Squamous Cell Carcinoma

* Pour 34 carcinomes épidermoïdes 


\begin{tabular}{|c|c|c|c|c|c|c|c|c|}
\hline & $\begin{array}{c}\mathrm{N} \\
\text { Patientes }\end{array}$ & Stade & $\begin{array}{c}\text { Dose et } \\
\text { fractionnement }\end{array}$ & Curiethérapie & $\begin{array}{c}\text { Reliquat } \\
\text { Histologique }\end{array}$ & $\begin{array}{l}\text { Rechute } \\
\text { locale à } \\
5 \text { ans }\end{array}$ & $\begin{array}{c}\text { Survie } \\
\text { Globale }\end{array}$ & $\begin{array}{l}\text { Survie } \\
\text { sans } \\
\text { rechute }\end{array}$ \\
\hline $\begin{array}{c}\text { Kirova } \\
\text { et al [10] }\end{array}$ & 70 & IB- II & $\begin{array}{c}36 \text { Gy en } 22 \\
\text { séances } \\
1,8 \text { Gy/séance }\end{array}$ & $24 \mathrm{~Gy}$ & $44 \%$ & $13 \%$ & $\begin{array}{l}77 \% \text { à } \\
3 \text { ans }\end{array}$ & \\
\hline $\begin{array}{l}\text { Lerouge } \\
\text { et al [11] }\end{array}$ & 42 & IB- II & $\begin{array}{l}\text { 40,5 Gy en } 18 \\
\text { séances } \\
2,25 \text { Gy/séance }\end{array}$ & $20 \mathrm{~Gy}$ & $48 \%$ & $10 \%$ & $\begin{array}{c}74 \% \text { à } \\
5 \text { ans }\end{array}$ & $\begin{array}{c}71 \% \text { à } \\
5 \text { ans }\end{array}$ \\
\hline $\begin{array}{l}\text { Huguet } \\
\text { et al [12] }\end{array}$ & 92 & IB- II & $\begin{array}{l}\text { 40,5 Gy en } 18 \\
\text { séances } \\
2,25 \text { Gy/séance }\end{array}$ & $20 \mathrm{~Gy}$ & $46 \%$ & $15 \%$ & $\begin{array}{c}78 \% \text { à } 5 \\
\text { ans }\end{array}$ & $\begin{array}{l}72,2 \% \\
\text { à } 5 \text { ans }\end{array}$ \\
\hline $\begin{array}{l}\text { Resbeut } \\
\text { et al [19] }\end{array}$ & 40 & $\begin{array}{c}\text { IB- II- III } \\
\text { IV }\end{array}$ & $\begin{array}{l}45 \text { Gy en } 25 \\
\text { séances } \\
1,8 \text { Gy/séance } \\
\text { bifractionné } \\
\text { pour } 20 \text { pts }\end{array}$ & $20 \mathrm{~Gy}$ & $51 \%$ & $8 \%$ & & $\begin{array}{l}66 \% \text { à } \\
2 \text { ans }\end{array}$ \\
\hline $\begin{array}{r}\text { Touboul } \\
\text { et al [21] }\end{array}$ & 150 & $\begin{array}{c}\text { IB- II- III } \\
\text { IV }\end{array}$ & $\begin{array}{l}45 \text { Gy en } 25 \\
\text { séances } \\
\text { 1,8 Gy/séance }\end{array}$ & $15 \mathrm{~Gy}$ & $52 \%$ & $12 \%$ & $\begin{array}{l}71 \% \text { à } \\
5 \text { ans }\end{array}$ & $\begin{array}{c}66 \% \text { à } \\
5 \text { ans }\end{array}$ \\
\hline $\begin{array}{l}\text { Notre } \\
\text { série }\end{array}$ & 45 & IB- II & $\begin{array}{l}\text { 40,5 Gy en } 18 \\
\text { séances } \\
2,25 \text { Gy/séance }\end{array}$ & $20 \mathrm{~Gy}$ & $36 \%$ & $4 \%$ & $\begin{array}{c}88 \% \text { à } 5 \\
\text { ans }\end{array}$ & $\begin{array}{c}85 \% \text { à } \\
5 \text { ans }\end{array}$ \\
\hline
\end{tabular}

$\underline{\text { Tableau 5. }}$. Séries d'association chimioradiothérapie avec chirurgie de clôture. 
F

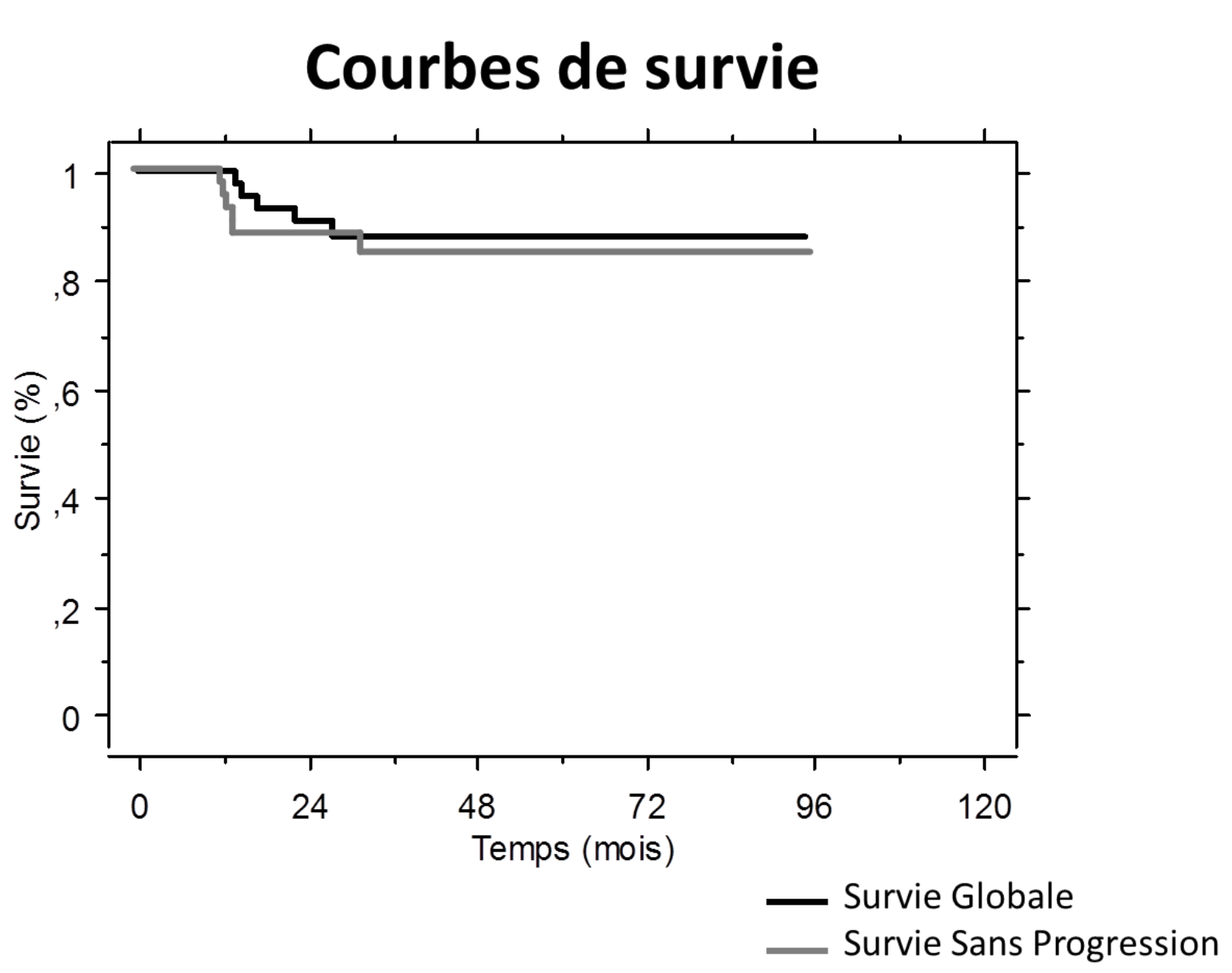

Fig 1. Courbes de Survie 


\section{Références}

1. Pecorelli S. Revised FIGO staging for carcinoma of the vulva, cervix, and endometrium. Int J Gynaecol Obstet 2009; 105: 103-104.

2. Gerbaulet AL, Kunkler IH, Kerr GR et al. Combined radiotherapy and surgery: local control and complications in early carcinoma of the uterine cervix--the Villejuif experience, 1975-1984. Radiother Oncol 1992; 23: 66-73.

3. Fyles AW, Pintilie M, Kirkbride $\mathrm{P}$ et al. Prognostic factors in patients with cervix cancer treated by radiation therapy: results of a multiple regression analysis. Radiother Oncol 1995; 35: 107-117.

4. Barillot I, Horiot JC, Pigneux $\mathrm{J}$ et al. Carcinoma of the intact uterine cervix treated with radiotherapy alone: a French cooperative study: update and multivariate analysis of prognostics factors. Int J Radiat Oncol Biol Phys 1997; 38: 969-978.

5. Perez CA, Grigsby PW, Chao KS et al. Tumor size, irradiation dose, and long-term outcome of carcinoma of uterine cervix. Int J Radiat Oncol Biol Phys 1998; 41: 307-317.

6. Chemoradiotherapy for Cervical Cancer Meta-Analysis Collaboration. Reducing uncertainties about the effects of chemoradiotherapy for cervical cancer: a systematic review and meta-analysis of individual patient data from 18 randomized trials. J Clin Oncol 2008; 26: $5802-5812$.

7. Morice P, Rouanet P, Rey A et al. Results of the GYNECO 02 study, an FNCLCC phase III trial comparing hysterectomy with no hysterectomy in patients with a (clinical and radiological) complete response after chemoradiation therapy for stage IB2 or II cervical cancer. Oncologist 2012; 17: 64-71. 
8. Keys HM, Bundy BN, Stehman FB et al. Cisplatin, radiation, and adjuvant hysterectomy compared with radiation and adjuvant hysterectomy for bulky stage IB cervical carcinoma. N Engl J Med 1999; 340: 1154-1161.

9. Houvenaeghel G, Lelievre L, Gonzague-Casabianca L et al. Long-term survival after concomitant chemoradiotherapy prior to surgery in advanced cervical carcinoma. Gynecol Oncol 2006; 100: 338-343.

10. Kirova YM, Bourhaleb Z, Alran S et al. [Preoperative concomitant radiochemotherapy in bulky carcinoma of the cervix: Institut Curie experience]. Cancer Radiother 2009; 13: 291297.

11. Lerouge D, Touboul E, Lefranc JP et al. [Preoperative concurrent radiation therapy and chemotherapy for operable bulky carcinomas of uterine cervix stages IB2, IIA, and IIB with proximal parametrial invasion]. Cancer Radiother 2004; 8: 168-177.

12. Huguet F, Cojocariu OM, Levy P et al. Preoperative concurrent radiation therapy and chemotherapy for bulky stage IB2, IIA, and IIB carcinoma of the uterine cervix with proximal parametrial invasion. Int J Radiat Oncol Biol Phys 2008; 72: 1508-1515.

13. ICRU. Dose and volume specification for intracavitary therapy in gynecology: ICRU Report 38. In Edition Bethesda, USA: 1985.

14. Piver MS, Rutledge F, Smith JP. Five classes of extended hysterectomy for women with cervical cancer. Obstet Gynecol 1974; 44: 265-272.

15. Narayan K, Hicks RJ, Jobling $\mathrm{T}$ et al. A comparison of MRI and PET scanning in surgically staged loco-regionally advanced cervical cancer: potential impact on treatment. Int J Gynecol Cancer 2001; 11: 263-271.

16. Boughanim M, Leboulleux S, Rey A et al. Histologic results of para-aortic lymphadenectomy in patients treated for stage IB2/II cervical cancer with negative 
[18F]fluorodeoxyglucose positron emission tomography scans in the para-aortic area. J Clin Oncol 2008; 26: 2558-2561.

17. Ramirez PT, Jhingran A, Macapinlac HA et al. Laparoscopic extraperitoneal paraaortic lymphadenectomy in locally advanced cervical cancer: a prospective correlation of surgical findings with positron emission tomography/computed tomography findings. Cancer 2011; 117: 1928-1934.

18. Chereau E, C DLH, Ballester M et al. The role of completion surgery after concurrent radiochemotherapy in locally advanced stages IB2-IIB cervical cancer. Anticancer Res 2013; 33: 1661-1666.

19. Resbeut M, Cowen D, Viens P et al. Concomitant chemoradiation prior to surgery in the treatment of advanced cervical carcinoma. Gynecol Oncol 1994; 54: 68-75.

20. Classe JM, Rauch P, Rodier JF et al. Surgery after concurrent chemoradiotherapy and brachytherapy for the treatment of advanced cervical cancer: morbidity and outcome: results of a multicenter study of the GCCLCC (Groupe des Chirurgiens de Centre de Lutte Contre le Cancer). Gynecol Oncol 2006; 102: 523-529.

21. Touboul C, Uzan C, Mauguen A et al. Prognostic factors and morbidities after completion surgery in patients undergoing initial chemoradiation therapy for locally advanced cervical cancer. Oncologist 2010; 15: 405-415.

22. Small W, Jr., Strauss JB, Jhingran A et al. ACR Appropriateness Criteria(R) definitive therapy for early-stage cervical cancer. Am J Clin Oncol 2012; 35: 399-405.

23. Eifel PJ, Winter K, Morris M et al. Pelvic irradiation with concurrent chemotherapy versus pelvic and para-aortic irradiation for high-risk cervical cancer: an update of radiation therapy oncology group trial (RTOG) 90-01. J Clin Oncol 2004; 22: 872-880. 
24. Nijhuis ER, van der Zee AG, in 't Hout BA et al. Gynecologic examination and cervical biopsies after (chemo) radiation for cervical cancer to identify patients eligible for salvage surgery. Int J Radiat Oncol Biol Phys 2006; 66: 699-705.

25. Vincens E, Balleyguier C, Rey A et al. Accuracy of magnetic resonance imaging in predicting residual disease in patients treated for stage IB2/II cervical carcinoma with chemoradiation therapy : correlation of radiologic findings with surgicopathologic results. Cancer 2008; 113: 2158-2165.

26. Ferrandina G, Petrillo M, Restaino G et al. Can radicality of surgery be safely modulated on the basis of MRI and PET/CT imaging in locally advanced cervical cancer patients administered preoperative treatment? Cancer 2012; 118: 392-403.

27. Keys HM, Bundy BN, Stehman FB et al. Radiation therapy with and without extrafascial hysterectomy for bulky stage IB cervical carcinoma: a randomized trial of the Gynecologic Oncology Group. Gynecol Oncol 2003; 89: 343-353.

28. Tewari KS, Sill MW, Long HJ, 3rd et al. Improved survival with bevacizumab in advanced cervical cancer. N Engl J Med 2014; 370: 734-743.

29. Renard-Oldrini S, Brunaud C, Huger S et al. [Dosimetric comparison between the intensity modulated radiotherapy with fixed field and Rapid Arc of cervix cancer]. Cancer Radiother 2012; 16: 209-214.

30. Gandhi AK, Sharma DN, Rath GK et al. Early clinical outcomes and toxicity of intensity modulated versus conventional pelvic radiation therapy for locally advanced cervix carcinoma: a prospective randomized study. Int J Radiat Oncol Biol Phys 2013; 87: 542-548.

31. Potter R, Haie-Meder C, Van Limbergen E et al. Recommendations from gynaecological (GYN) GEC ESTRO working group (II): concepts and terms in 3D imagebased treatment planning in cervix cancer brachytherapy-3D dose volume parameters and 
aspects of 3D image-based anatomy, radiation physics, radiobiology. Radiother Oncol 2006; 78: 67-77.

32. Klopp AH, Eifel PJ. Biological predictors of cervical cancer response to radiation therapy. Semin Radiat Oncol 2012; 22: 143-150. 